\title{
New methodology for modeling, design and implementation of RF power amplifiers
}

\author{
Guillermo Rafael-Valdivia, Omar Castellanos Ballesteros. \\ Universidad Tecnológica del Perú. UTP, Arequipa, Perú. c16699@utp.edu.pe \\ Universidad Estatal Península de SantaElena, Ecuador.ocastellanos@upse.edu.ec
}

\begin{abstract}
This work presents a new methodology for modeling, design and implementation of power amplifiers in different technologies. As result of comparison, a flowchart with a new methodology is proposed which can be useful for the designer to design and implement power amplifiers with low, medium and high power devices in different technologies. This paper is divided in 4 parts: The first one is an introduction to the importance of modeling and design techniques in the final implementation of power amplifiers for the modern communication systems. The second one details the modeling process for different technologies, which final result is a unified model. The third part is related with the characterization of high power transistors, with special emphasis on substrate characterization and final implementation of a power amplifier. Finally, in the fourth part, the new methodology is proposed based on the comparisons of previous procedures.
\end{abstract}

Index Terms - microwaves, modeling, power amplifier, substrate.

\section{INTRODUCTION}

Modern communication systems require RF power amplifiers to operate with large signal in broadband conditions. For $5 \mathrm{G}$ systems, requirements will be more demanding; and different technologies such as GaN and LDMOS are currently under investigation. Those two technologies have improved power density compared with traditional compounds semiconductors [1]. It originates problems with power dissipation which creates dispersion and thermal effects [2]. Consequently, for the design of RF power amplifiers using these semiconductors, it is essential to use accurate models that take into account these phenomena. Furthermore, for power amplifier implementation, an accurate substrate characterization is required, in order to get the appropriate input and output impedances for the matching networks. In this paper we will show that through the use of models flexible enough to predict static and dynamic conditions, and a one-port substrate characterization technique, we can demonstrate that it is possible to implement efficiently RF power amplifiers in LDMOS technologies. Based on those steps we propose a new methodology which is summarized in Fig. 1. That methodology will be explained in the following sections. 


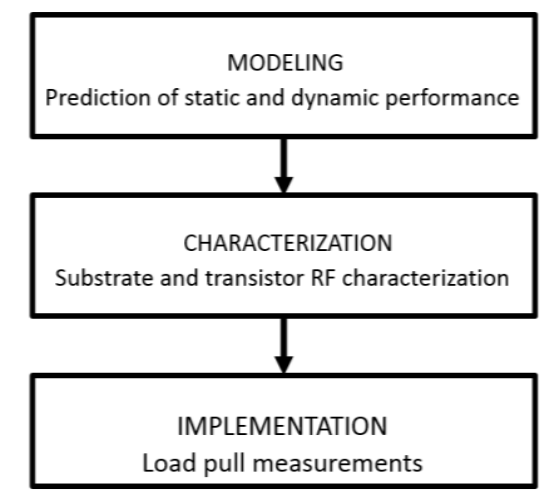

Fig. 1. Flow chart of the proposed methodology for the power amplifier design.

\section{MODELING}

Modern communication systems, such us internet of things (IoT) and 5G are currently under investigation; however they will demand the use of different technologies for amplifiers. For instance GaAs is a promising technology for low power and linear applications; and GaN / LDMOS for high power amplifiers for long distance communications. As these 2 technologies behave differently, it makes sense to consider different strategies for modeling. However despite their differences, there are some common characteristics. One of them is the output conductance (gds) and transconductance (gm) frequency dispersion, considered as one of the causes for the memory effects which cause complexity in linearization [3]. In this paper we will call this effect "the frequency dispersion phenomena". We characterized this effect through I/V pulsed measurements by using the DIVA system provided by Accent Technologies [4]. The measurements were carried out in University College Dublin in the RF and microwave group. As it is shown in Fig. 2, DIVA system is a 2 port equipment, which is able to send and read pulsed voltages in the drain and gate side of a microwave transistor placed in a specific test fixture.

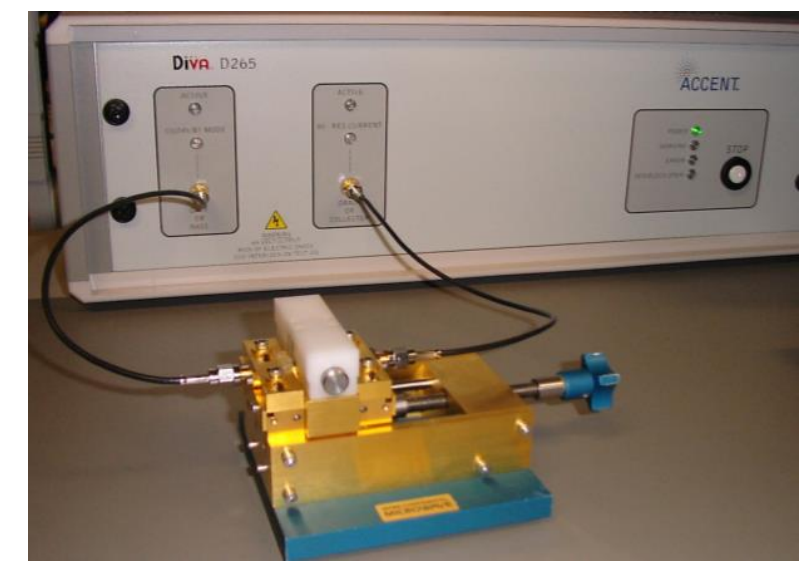

Fig. 2. Diva Measurement system used to characterize the frequency dispersion phenomena. The transistor under test is located below the plastic pusher in the test fixture. 
In Fig. 3 it is shown the RF signal sent by DIVA which has a pulse width of 0.2 usec with a period about $1 \mathrm{~ms}$. This duty cycle allows to measure only the dynamic behavior by keeping the same bias point. The system can measure the static (DC) characteristics of any device and compare them with the dynamic (pulsed) characteristics.

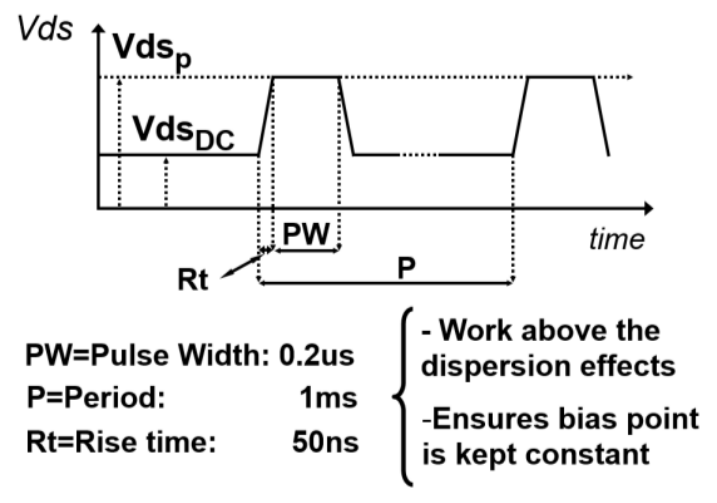

Fig. 3. Voltage waveform at the drain side of a transistor, provided by the DIVA pulsed measurement test system.

For a NE76038 GaAs low power HEMT, the evidence of the frequency dispersion effect is shown in Fig. 4. We can see difference in the slopes of the IV curves for the static and dynamic conditions. [5]. Furthermore it is possible to set different DC bias points (multibias) and perform pulsed measurements for each case. In this scenario there are also differences between each of the pulsed characteristics. It means that the difference in the slopes are due not only to the bias points positions; but also to the trapping effects as it is indicated in Fig. 5.

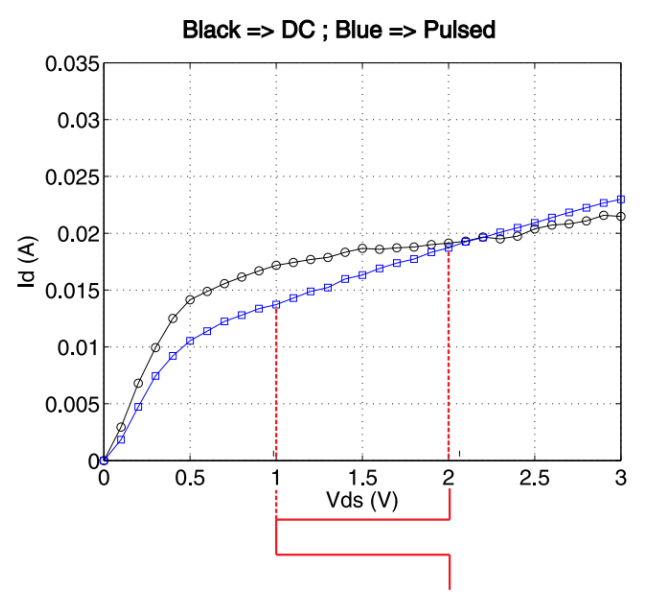

Fig. 4. DC and Pulsed IV characteristics for the NE76038 GaAs HEMT device.

In order to demonstrate that by using only a single non linear Ids equation we can predict all of these effects, we used the technique proposed in [4]. In that technique we demonstrated that by using COBRA model [6] we could predict the DC measurements. However in order to include in that model the dynamic behavior of the device, we used the effective voltages technique and modified the large 
signal equivalent circuit. In fact, this technique uses the pulsed voltages as inputs in the effective voltages equations in order to provide the proper argument for the Ids non-linear equation [4]. The effective voltages equations are shown in (1) and (2), where $\mathrm{vds}_{\mathrm{e}}$ and $\mathrm{vgs}_{\mathrm{e}}$ are the effective voltages, $\mathrm{d}_{\mathrm{vd}}$ and $\mathrm{d}_{\mathrm{vg}}$ are the pulsed voltages, and $\mathrm{k}_{\mathrm{i}}$ are fitting parameters.

$$
\begin{aligned}
& \mathrm{vdSe}=\mathrm{d}_{\mathrm{vd}} \cdot\left(\mathrm{k}_{1}-\mathrm{k}_{2} \cdot \mathrm{d}_{\mathrm{vd}}\right)+\mathrm{Vdi}_{\mathrm{DC}}+\mathrm{d}_{\mathrm{vg}} \cdot\left(\mathrm{k}_{3}-\mathrm{k}_{4} \cdot \mathrm{d}_{\mathrm{vg}}\right) . \\
& \mathrm{vgSe}=\mathrm{d}_{\mathrm{vg}} \cdot\left(\mathrm{k}_{5}-\mathrm{k}_{6} \cdot \mathrm{d}_{\mathrm{vg}}\right)+\mathrm{Vgi}_{\mathrm{DC}}+\mathrm{d}_{\mathrm{vd}} \cdot\left(\mathrm{k}_{7}-\mathrm{k}_{8} \cdot \mathrm{d}_{\mathrm{vd}}\right) .
\end{aligned}
$$

The pulsed voltages are extracted from the large signal equivalent circuit by using a four-terminal topology which is shown in Fig. 6.
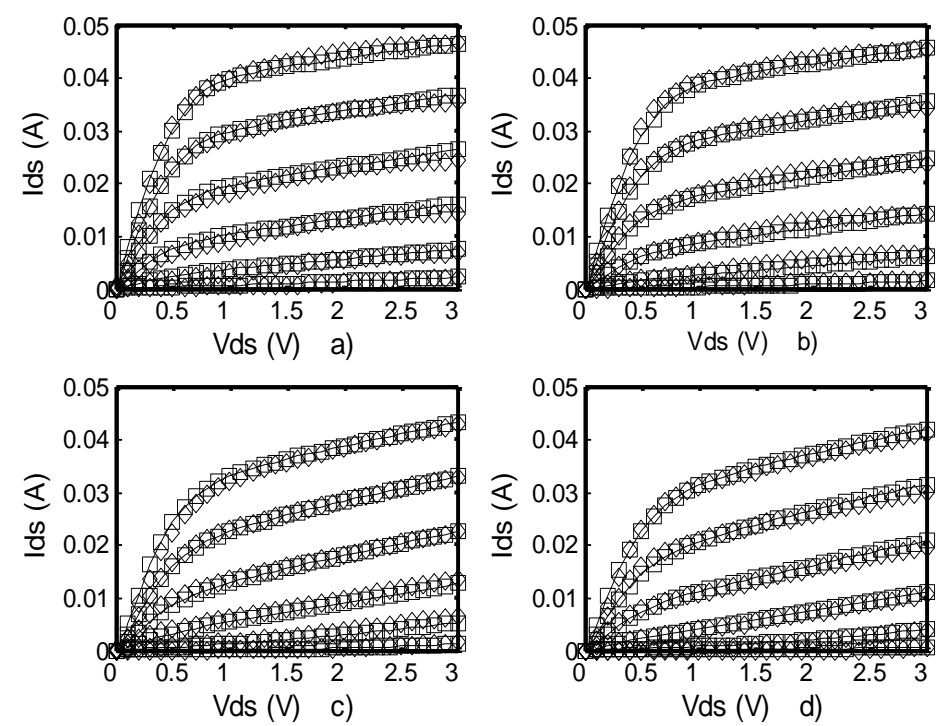

Fig. 5. Plot of the measured (diamonds) and modeled (squares) pulsed drain to source current obtained for the NE76038 with the proposed technique. Pulse width $0.2 \mu \mathrm{sec}$ and duty cycle: $0.02 \%$. Extrinsic applied voltages: a) $\mathrm{Vgs}_{\mathrm{DC}}=0 \mathrm{~V}, \mathrm{Vds}_{\mathrm{DC}}=0.5 \mathrm{~V}$;

b) $\mathrm{Vgs}_{\mathrm{DC}}=-1 \mathrm{~V}, \mathrm{Vds}_{\mathrm{DC}}=0.5 \mathrm{~V}$; c) $\mathrm{Vgs}_{\mathrm{DC}}=0 \mathrm{~V}, \mathrm{Vds}_{\mathrm{DC}}=3 \mathrm{~V}$; d) $\mathrm{Vgs}_{\mathrm{DC}}=-1 \mathrm{~V}, \mathrm{Vds}_{\mathrm{DC}}=3 \mathrm{~V}$. Sweep in gate voltage: $0 \mathrm{~V}$ to $-1 \mathrm{~V}$ (uniform step). Similar accuracy was obtained in multibias conditions. [4]

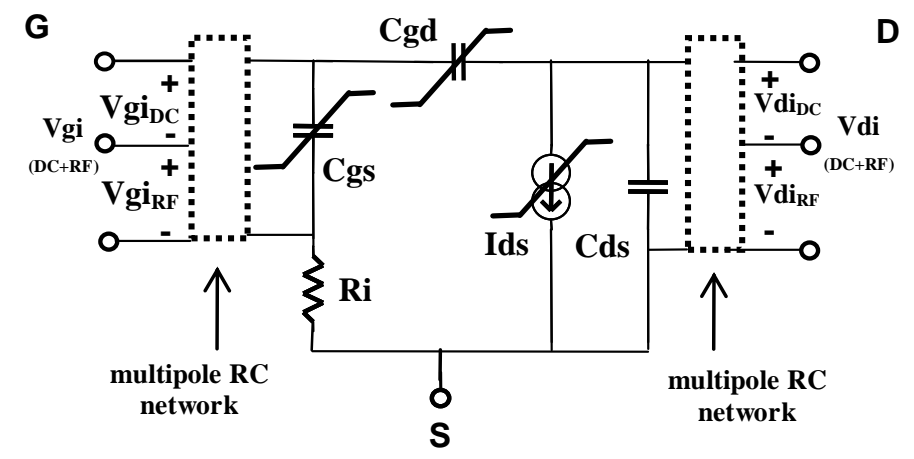

Fig. 6. Proposed non linear large signal equivalent circuit for microwave transistors. 
For a CGH40010F 10W GaN HEMT transistor, we repeated the same test; but keeping the same bias point and changing only the pulse width. For this case, Angelov nonlinear equation for Ids [2] was implemented. By applying the same effective voltage technique explained for the GaAs transistor, we got a new model in which the dynamic equation was embedded within the Angelov nonlinear equation for Ids. Results are shown in Fig. 7.
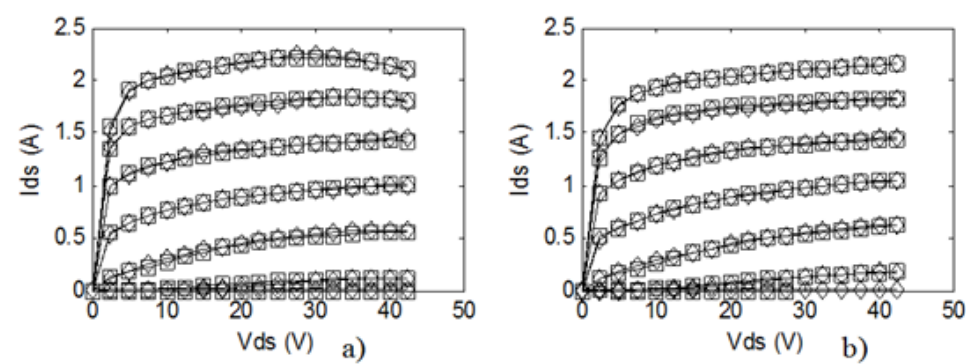

Fig. 7. Measured (diamonds) and modeled (squares) pulsed Ids obtained $@ \mathrm{Vgs}_{\mathrm{DC}}=-4 \mathrm{~V}, \mathrm{Vds}_{\mathrm{DC}}=40 \mathrm{~V}$ for the CGH40010F 10W HEMT transistor. a) Pulse width: $30 \mu \mathrm{sec}$. b) Pulse width: $5 \mu \mathrm{sec}$.

One of the properties of this model is that it can be used to model the RF performance. In Fig. 8 it is shown the difference between modeled and measured S-parameters. Furthermore, with the complete characterization of the device, the proposed model can be used to predict the output power vs input power behavior as it is shown in Fig. 9 .

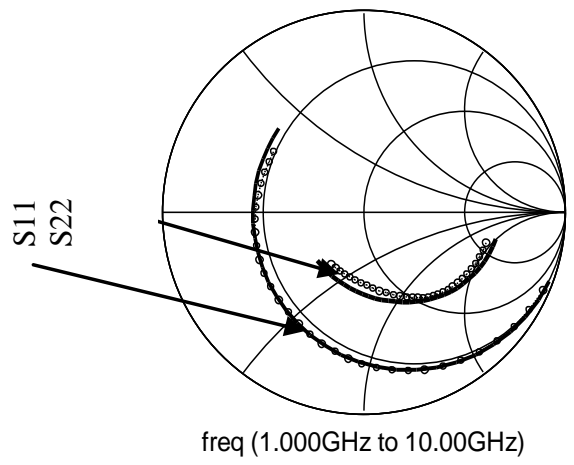

Fig. 8. Measured (circles) and modeled (lines) S-parameters for the NE76038 using the proposed method. Extrinsic applied voltages: $\mathrm{Vds}_{\mathrm{DC}}=3 \mathrm{~V} ; \mathrm{Vgs}_{\mathrm{DC}}=0 \mathrm{~V}$. Similar agreement was obtained in multibias conditions and for other S-parameters.

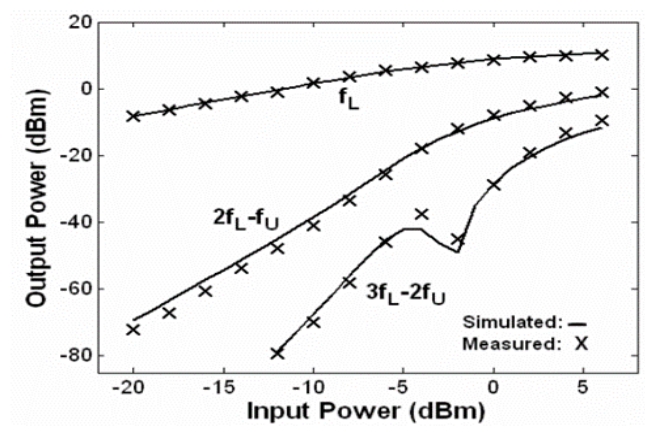

Fig. 9. Large signal two tone output power performance centered at $2 \mathrm{GHz}$ and tone spacing of $4 \mathrm{MHz}$ at $\mathrm{Vds}_{\mathrm{DC}}=2 \mathrm{~V}$ and $\mathrm{VgS}_{\mathrm{DC}}=-0.2 \mathrm{~V}$ 
In conclusion for this section, we can say that despite the different non-linear equations for Ids used for the GaAs and GaN devices (COBRA and Angelov respectively), the same methodology for modeling was used and validated. This methodology provided us coherent predictions for the static and dynamic behavior for both technologies.

\section{SUBSTRATE CHARACTERIZATION AND POWER AMPLIFIER IMPLEMENTATION}

After finishing the modeling process, we focus on the substrate characterization and the power amplifier implementation procedures. Both of them have been carried out in our university. In this section we will demonstrate that it is possible to build power amplifiers according to specific target specs using low cost equipment but well stablished models. Finally we will compare the results with the ones shown in datasheets showing coherence.

\section{A. Substrate characterization}

Before to do the design and implementation of an amplifier, the designer requires to know the dielectric constant and loss tangent values of the substrate to be used. Normally the designer uses the values provided in the datasheets which are average values dependent on the fabrication process. However, when the complexity of the circuit requires high accuracy, substrate characterization is a must. (i.e. power amplifiers in microwave ranges). Several methods have been proposed to measure the fundamental properties of substrates. Some of them are based on physical measurements in low frequencies using the relationship between the capacitance of a dielectric, the permittivity and the thickness of the dielectric [7]. However their accuracy is not enough for high frequency conditions (GHz range). Furthermore with that kind of methods is not possible to get loss tangent values. In [8], the methods for measuring permittivity are summarized in 2 categories: resonant and non-resonant. In some telecommunications companies, such as Freescale Semiconductors (now NXP), according to the professional experience of the author, the resonant two-port characterization technique is mainly used for power amplifier design. This technique is based on the measurements of scattering parameters, specifically the transmission parameter S21. That method allows the measurement of the transmission coefficient at resonant frequencies in order to use those results as reference values, in order to find the dielectric constant $(\varepsilon)$ and losses $(\tan \delta)$ through optimization procedures performed by CAD tools. This method is accurate enough; but it requires the use of a 2 port equipment such a Vector Network Analyzer (VNA), which sometimes is not available in public universities in our country. In this section, we will show that using only return loss measurements in a single port configuration it is possible to characterize substrates with accuracy in the microwave range. The advantage of this technique is its simplicity and accuracy. Furthermore, in environments with lack of resources, it is a low cost solution because the use of VNA is not required. The proposed method in this paper for substrate characterization begins with the design of an open shunt stub resonator, as it is shown in Fig. 
10. We used as test vehicle the low cost FR-4 substrate. The shunt stub is a quarter wave length line at a specific design frequency. It presents a variable impedance to the access ports. So, according to the frequency of the excitation signal, the impedance of the stub can change from open to short. So we will have minimum or maximum values of S11 and S21 at specific resonant frequencies. In our design we decided to set the first resonance frequency at $600 \mathrm{MHz}$ (design frequency). We have selected that value considering that its second harmonic must be within the frequency range of our spectrum analyzer (HP8591A). Previous to the measurements, we compared the behavior of the simulated S11 and $\mathrm{S} 21$ for our resonator as it is shown in Fig 11.

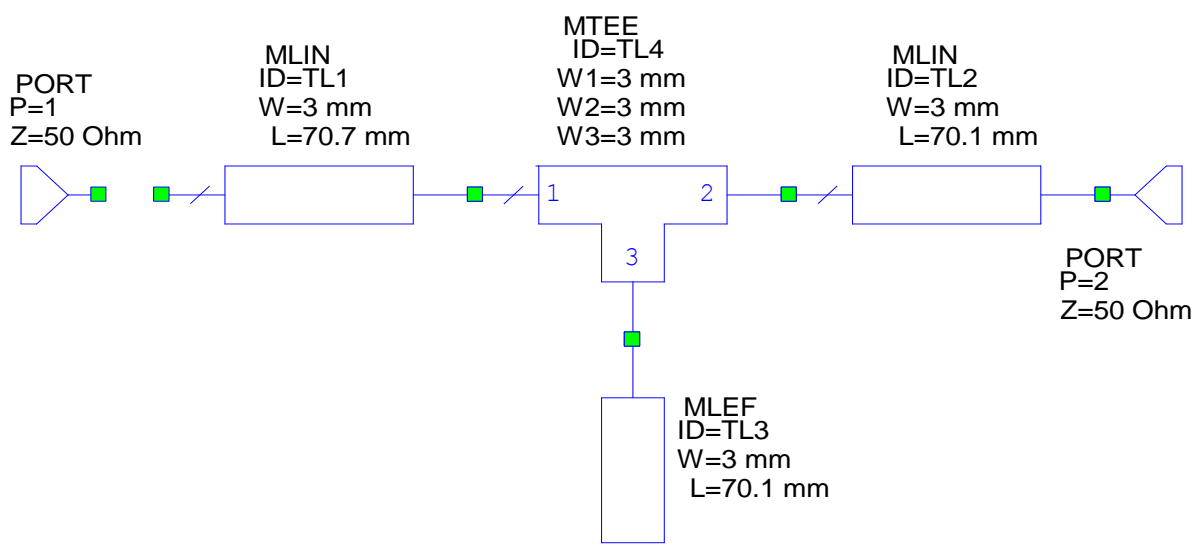

Fig. 10. Proposed resonator topology for substrate characterization. Port 2 can be connected to a $30 \mathrm{~dB}$ attenuator.

In Fig. 11 it is possible to appreciate that for the conventional method (S21 measured with VNA) the first local minimum of S21 is at the design frequency $(600 \mathrm{MHz})$. However for the proposed method (S11 measured only with a spectrum analyzer and a coupler), the first local minimum of S11 is at the second harmonic $(1200 \mathrm{MHz})$. We have verified by using the optimization tools of AWR (Microwave Office), that variations on $\varepsilon$ are responsible for shifting the resonant frequency (horizontal variation in local minimum of S11), while tuning on $\tan \delta$ have direct impact to determine how deep is the resonance valley in S11 (vertical offset). The influence of the optimization of the dielectric constant and the loss tangent are independent (they don't impact to each other). Consequently, through an optimization of $\varepsilon$ and $\tan \delta$ it is possible to fit S11 simulations with S11 measurements, so it is possible to find the actual value of the substrate parameters. In Fig. 11 it is possible to see that an advantage of the proposed 1-port method, over the conventional one, is the presence of a local maximum, located at the central frequency fc (close to $0.6 \mathrm{GHz}$ ). This point is used as reference to check the $\varepsilon$ value; and to determine the final value of $\tan \delta$. 


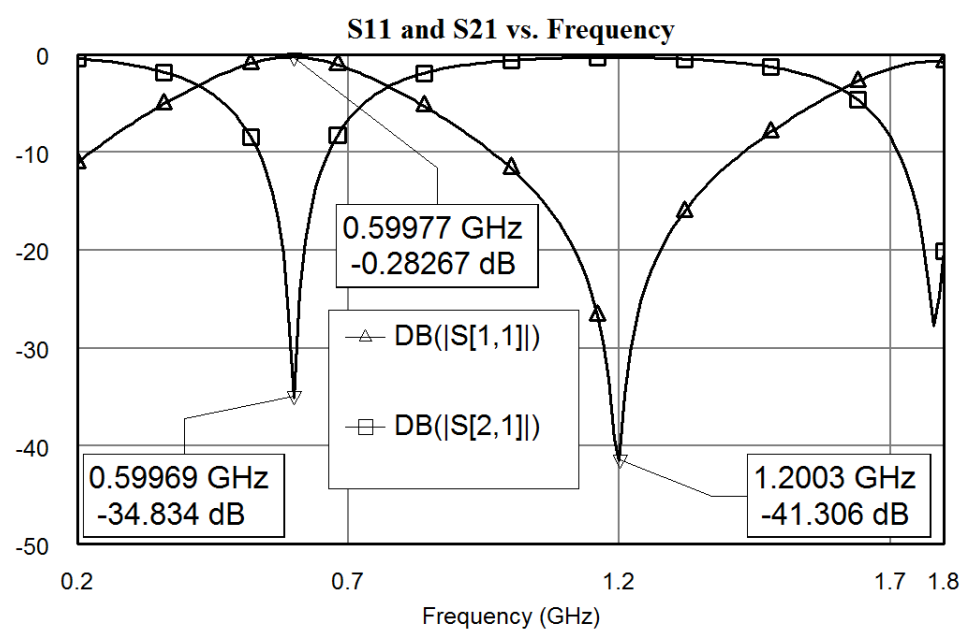

Fig. 11. Comparison between the behavior of simulated S11 and S22 for substrate characterization purposes

To perform the measurements, the setup shown in Fig.12 was implemented, where SUT is the substrate under test; Pref represents the reflected power; Pinc the incident power; Pmeas represents the measured power; CF represents the coupling factor; and RL the return loss.

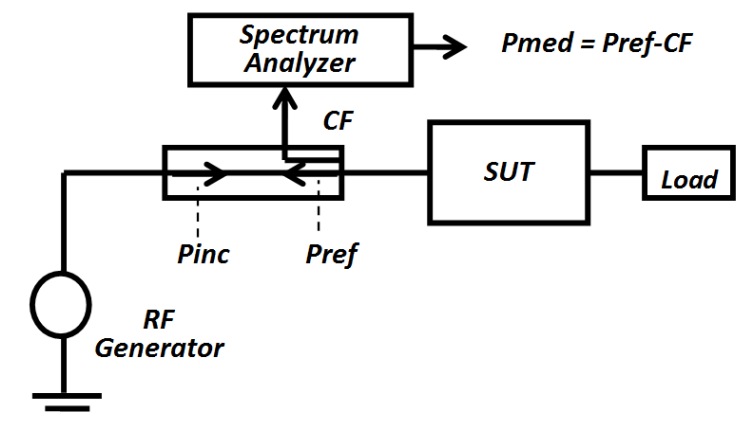

Fig. 12. Block diagram of the proposed method for the 1-port substrate characterization.

The coupling factor and insertion loss were measured in the frequencies of interest. A Rohde and Schwarz RF (SMT06) signal generator has been programmed in frequency sweep mode from $200 \mathrm{MHz}$ until $1.8 \mathrm{GHz}$, covering the previously defined central frequency (fc) and its second harmonic. Those values are within the range of operation of the Spectrum Analyzer HP8591A. As it is shown in Fig. 13, experimentally we have seen the resonant frequency of Pmed at $1158 \mathrm{MHz}$. Furthermore, there is a maximum at $579 \mathrm{MHz}$. Both of them are a clear effect of the substrate (resonator) connected to the setup. The second minimum shown in Fig. 13 at $1455 \mathrm{MHz}$, is due to the cutoff frequency of the coupler. From those results, the return loss of the substrate under test is calculated according to: 


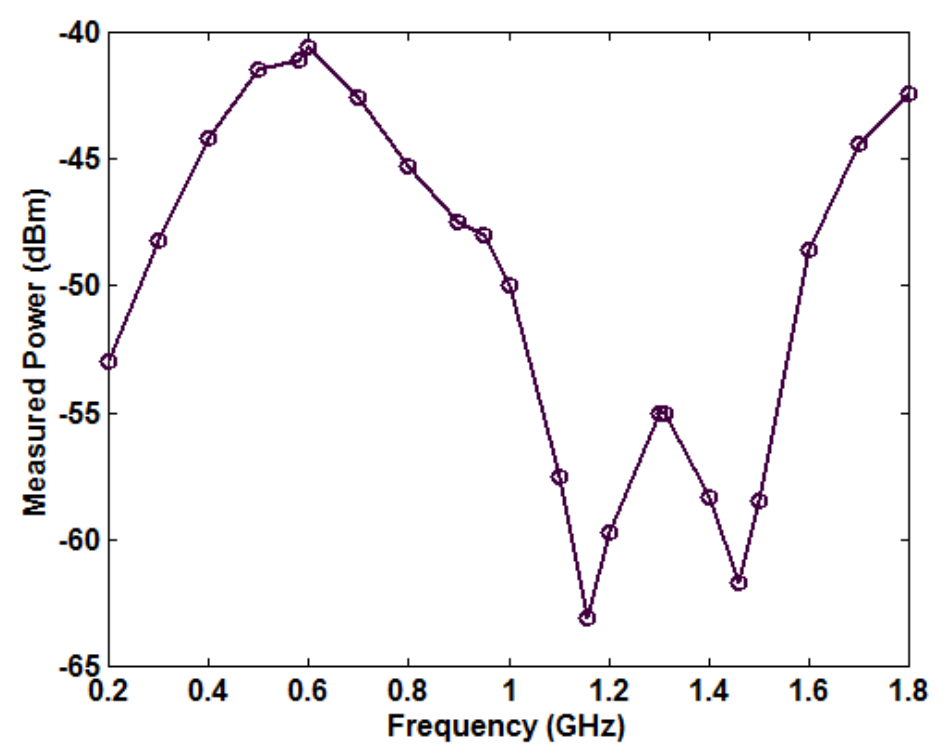

Fig. 13. Measured power (Pmeas or Pmed), using the setup shown in Fig. 12.

Consequently, those values of Return Loss (RL) can be compared with simulated results, as shown in figure 14. Considering that the geometrical tolerance in the implementation of the circuit (dimension of the lines) is accurate enough, the difference between measurements and simulations is only due to the physical parameters of the substrate: $\varepsilon$ and $\tan \delta$.

From initial simulations in Microwave Office, the maximum was located at $600 \mathrm{MHz}$ and the minimum at $1200 \mathrm{MHz}$. So, the parameter $\varepsilon$ was optimized in simulations, shifting the resonance frequency until the peak reaches the measured value: $1158 \mathrm{MHz}$. By doing this, the position of the local maxima was automatically placed at its measured value: $579 \mathrm{MHz}$, confirming the coherence of the method. Once the frequencies were aligned, we had to deal with the magnitude values of return loss. To do this, $\tan \delta$ was optimized fitting the local maximum magnitude. It is important to remark that doing this second optimization, there is no impact in the position of the resonant frequencies. The differences in the deep zone of the curves are expected, and are due to the low magnitude of measured power at those points and to the resolution of the instrument. 


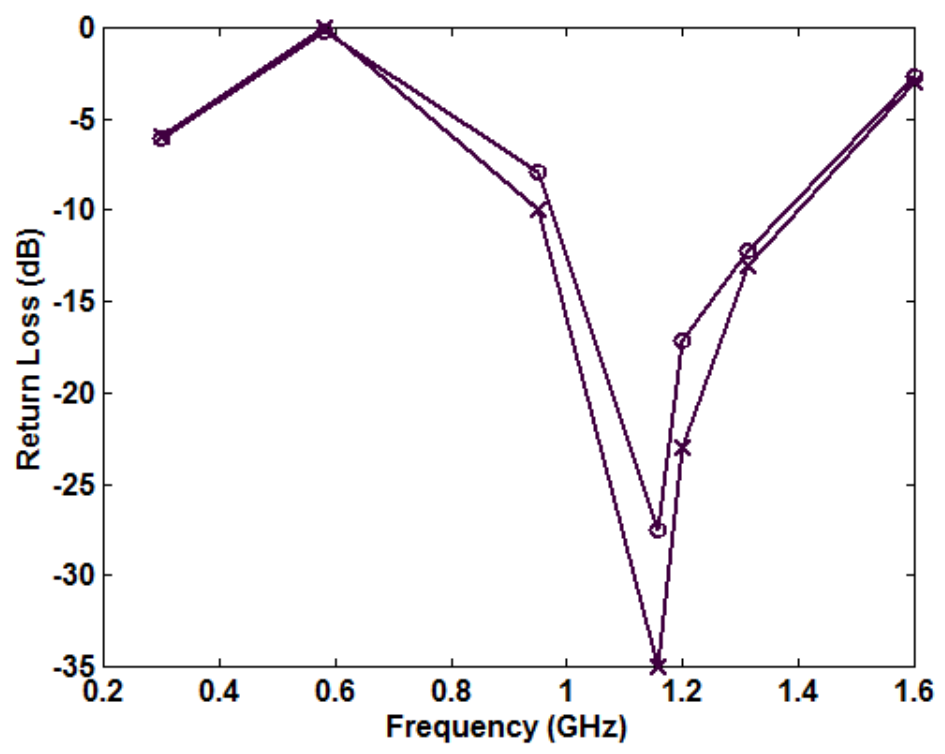

Fig. 14. Simulated (x) and measured (o) return loss for the substrate under test.

After the optimization process, we got $\varepsilon=4.4$ and $\tan \delta=0.02$. The average values for these parameters, indicated in [9] are: $\varepsilon=4.7$ and $\tan \delta=0.014$. Those values are also coherent with the ones indicated in [10]. In order to confirm the validity of the proposed method, simulations in ADS were performed as well. The results obtained are coherent with the ones that we got through optimization in Microwave Office. The same characterization process was performed with the RF35 substrate; we got $\varepsilon=3.5$ and $\tan \delta=0.002$. The thickness of the substrate was 20 mils $(0.5 \mathrm{~mm})$. The average values for these parameters, indicated in [11] are: $\varepsilon=3.5$ and $\tan \delta=0.0018$. Fig. 15 shows the resonator implemented to characterize the RF-35 substrate.

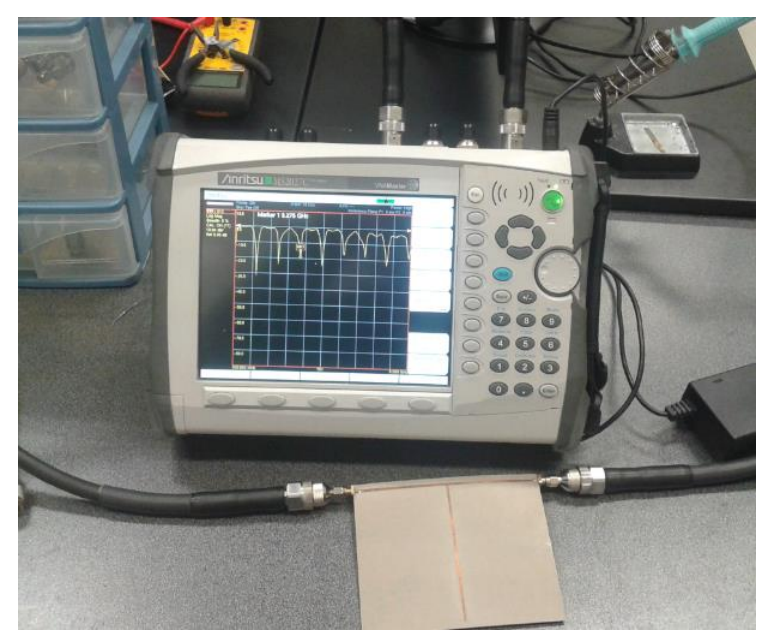

Fig. 15. Resonator used to characterize the RF35 substrate 


\section{B. Power Amplifier Implementation}

We built a power amplifier using the MRF6S18100 LDMOS device from Freescale Semiconductors (now NXP). We used the RF35 substrate previously characterized with the parameters and dimensions shown in the previous paragraph. According to its datasheet given in [12], this field effect transistor was designed for base station applications, from 1800 to $2000 \mathrm{MHz}$ with 100 Watts output power and $15 \mathrm{~dB}$ gain (at $\mathrm{P} 1 \mathrm{~dB}$ conditions). The recommended bias point for this device is $\mathrm{VDD}=28$ Volts (typical for LDMOS) at IDQ $=900 \mathrm{~mA}$ (class $\mathrm{AB}$ ). In contrast to the conventional bias tee configuration with discrete inductance and capacitors, the bias network in our design uses a quarter wavelength line with a shunt cap in order to provide a virtual RF short circuit at a quarter wave distance from the drain terminal of the transistor. Similar procedure was used to bias the gate side. For both cases we used symmetrical feeders in order to improve bandwidth. Simulations of the bias network design are shown in Fig. 16.
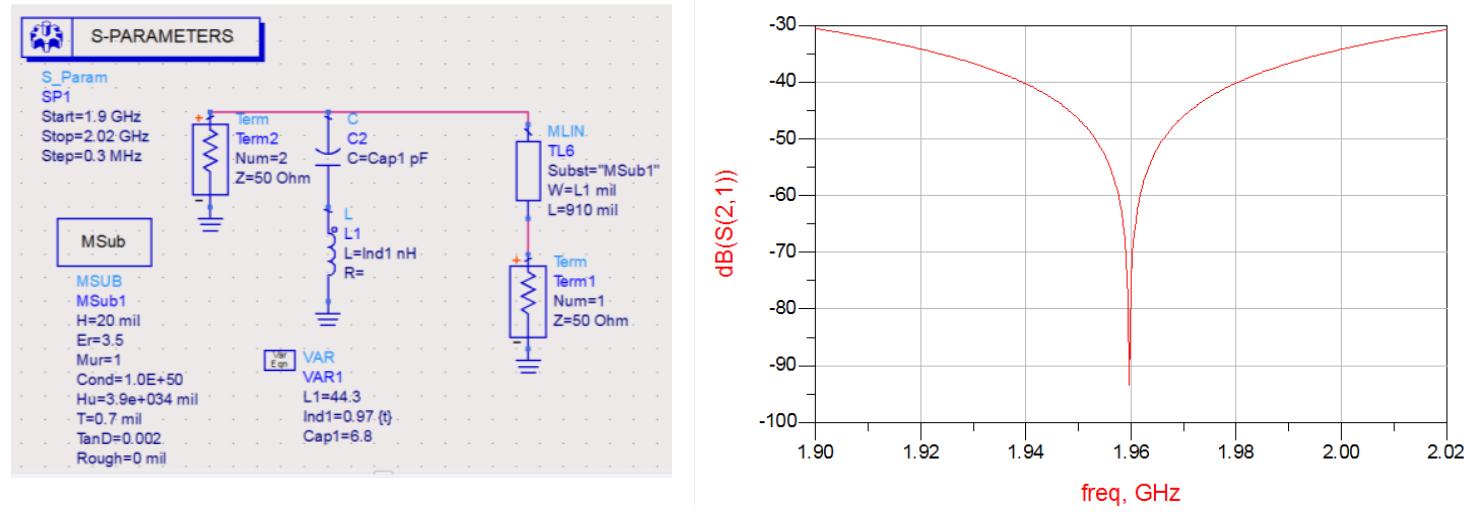

Fig. 16. Bias network simulations for the power amplifier based on the MRF6S18100 LDMOS device.

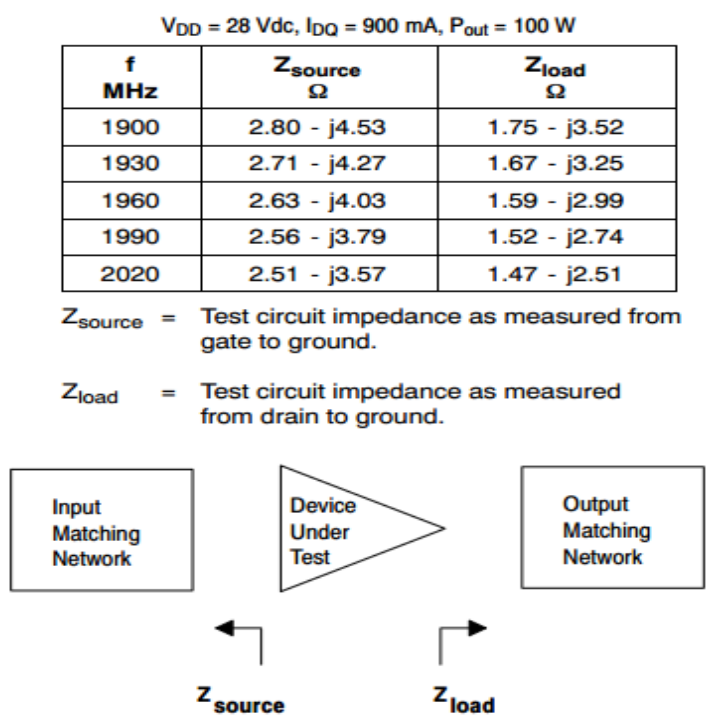

Fig. 17. Source and load impedances extracted from load pull measurements for the MRF6S18100 LDMOS device [12]. 
For the design process of the matching networks, instead of using the conventional lumped or discrete matching narrow band techniques optimized only for power or gain; we used wideband techniques with the combination of lumped and discrete components, in order to take advantage of the best properties of each technique. The goal of those networks are to allow the transistor to see the same impedances shown in the load pull data, depicted in Fig.17 in order to meet the target specs (best trade-off between power, efficiency and linearity).

The input and output matching networks are shown in Figs. 18 and 19 respectively, in which the bypass capacitor (modeled as a capacitor in series with a parasitic inductance of $0.9 \mathrm{nH}$ ) is not shown for visualization purposes of the trajectory of the impedances (Fig. 20 and 21). Its effect is small because it acts like a short circuit at the central frequency. Furthermore, we can see that the impedances obtained in our design (Table I) are similar to the ones provided by the data sheet.

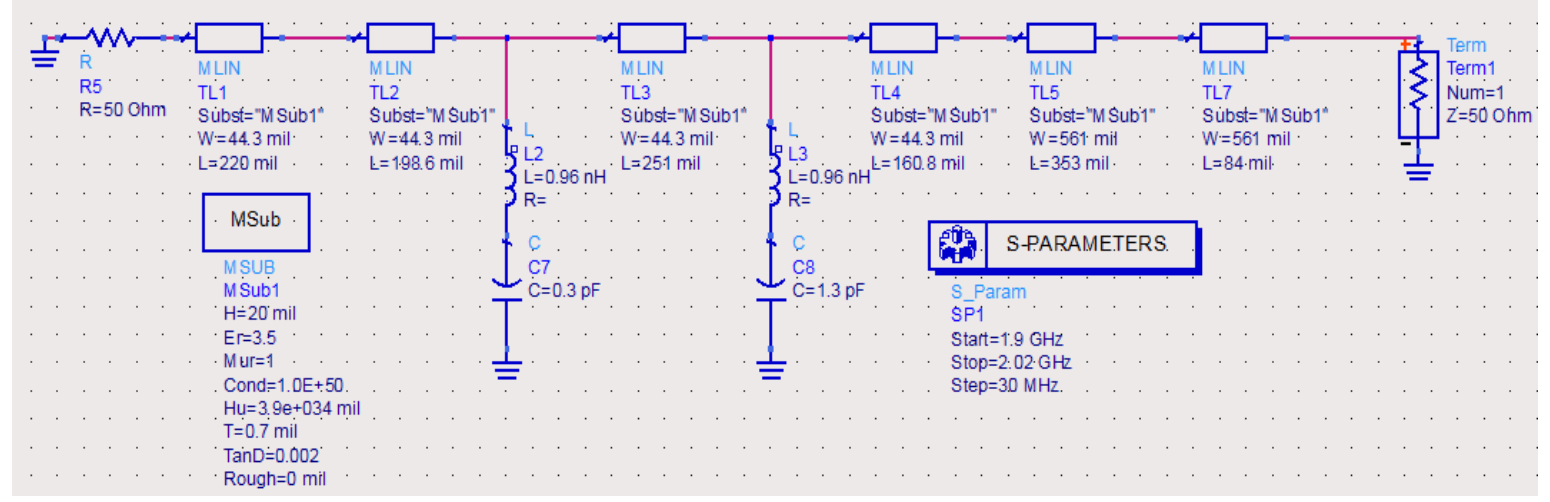

Fig. 18 Input matching network for the power amplifier based on the MRF6S18100 LDMOS device.

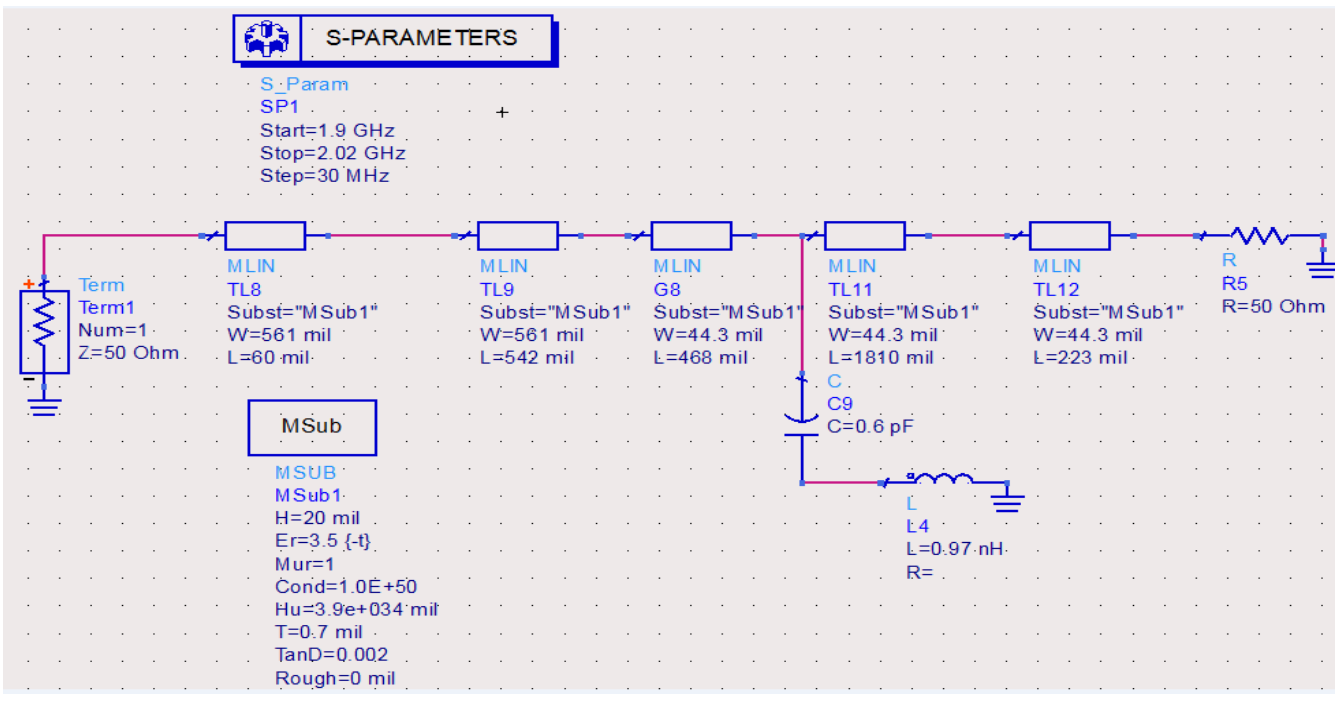

Fig. 19. Output matching network for the power amplifier based on MRF6S18100 LDMOS device. 
Journal of Microwaves, Optoelectronics and Electromagnetic Applications, Vol. 16, No. 3, September 2017797 DOI: http://dx.doi.org/10.1590/2179-10742017v16i3955

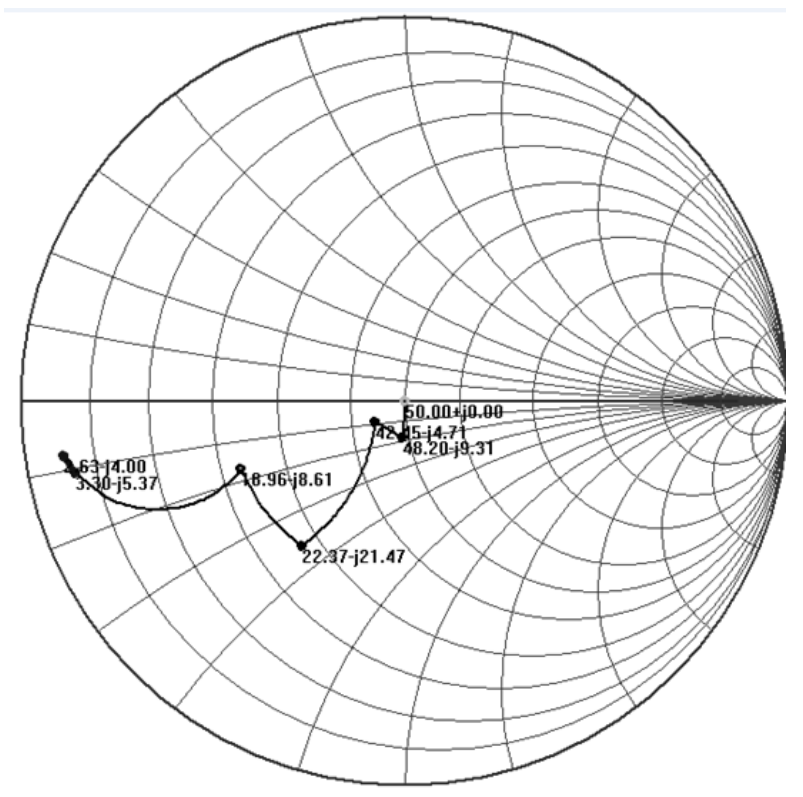

Fig. 20. Trajectory of the impedances for the input matching network for the power amplifier based on the MRF6S18100 LDMOS device.

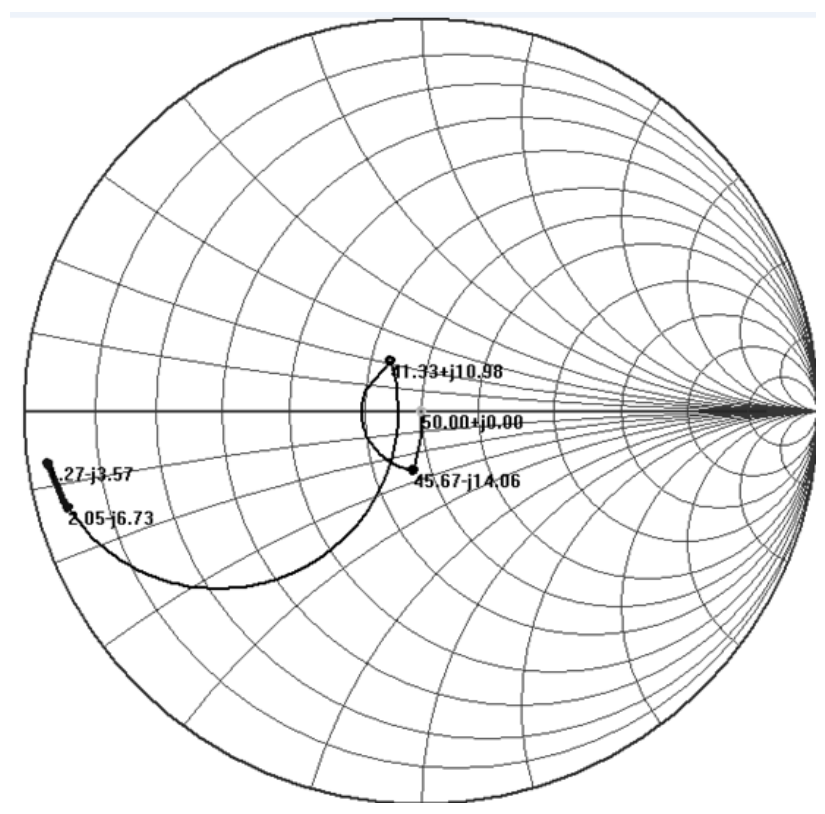

Fig. 21. Trajectory of the impedances for the output matching network for the power amplifier based on the MRF6S18100 LDMOS device. 
Journal of Microwaves, Optoelectronics and Electromagnetic Applications, Vol. 16, No. 3, September 2017798

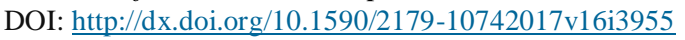

TABLE I. SOURCE AND LOAD IMPEDANCES OBTAINED FOR THE FINAL POWER AMPLIFIER DESIGNED WITH THE MRF6S18100 LDMOS DEVICE

\begin{tabular}{ccc}
\hline Frequency (MHz) & Zsource (ohms) & Zload (ohms) \\
\hline 1900 & $2.68-\mathrm{j} 4.33$ & $1.48-\mathrm{j} 3.25$ \\
1930 & $2,65-\mathrm{j} 4,18$ & $1.51-\mathrm{j} 3.13$ \\
1960 & $2,64-\mathrm{j} 4,02$ & $1.54-\mathrm{j} 3.03$ \\
1990 & $2,63-\mathrm{j} 3,87$ & $1.55-\mathrm{j} 2.95$ \\
2020 & $2,62-\mathrm{j} 3,72$ & $1.56-\mathrm{j} 2.87$ \\
\hline
\end{tabular}

To test the performance of our amplifier in different conditions, we performed a power sweep test in three different frequencies in order to extract the gain and capture the output power at $\mathrm{P} 1 \mathrm{~dB}$ conditions. Our system calculates the gain from every measured power. It stops when the calculated gain compresses $1 \mathrm{~dB}$. This allow us to capture the output power at $\mathrm{P} 1 \mathrm{~dB}$, whose values are shown in Table II. We selected 3 frequencies: 1930, 1960 and 1990MHz, because this is the frequency band indicated in the data sheet of our transistor [12] (page 1) for its specific application. By doing that, we characterized the amplifier in the edges and in its central frequency. As it is shown in Table II, the obtained values of those parameters agree with the values indicated in the data sheet for the targeted frequency band.

TABLE II. POWER SWEEP MEASUREMENTS FOR THE FINAL POWER AMPLIFIER DESIGNED WITH THE MRF6S18100 LDMOS DEVICE

\begin{tabular}{crrrrr}
\hline Freq(MHz) & G(dB) & Pin(dBm) & Pout $(\mathbf{d B m})$ & Pout(W) & IRL(dB) \\
\hline 1930.00 & 14.56 & 37.00 & 50.53 & 113.06 & -18.34 \\
1960.00 & 14.66 & 37.01 & 50.66 & 116.36 & -30.37 \\
1990.00 & 14.67 & 36.96 & 50.63 & 115.57 & -16.41 \\
\hline
\end{tabular}

The measured average gain and ripple were $14.63 \mathrm{~dB}$ and $0.11 \mathrm{~dB}$ respectively. Finally we performed a frequency sweep test in small signal conditions $(1 \mathrm{CW})$ in order to obtain the global behavior of the amplifier. As it is shown in Fig. 22, we got less than $0.2 \mathrm{~dB}$ gain flatness from 1930 MHz until $2000 \mathrm{MHz}$, with IRL values better than $-14.5 \mathrm{~dB}$. The final implemented amplifier is shown in Fig. 23. The transistor is in the middle of the PCB. This device has been measured with plastic pushers attached with screws (not shown in the picture) in order to avoid soldering and to test the device in different PCBs and compare performances. 


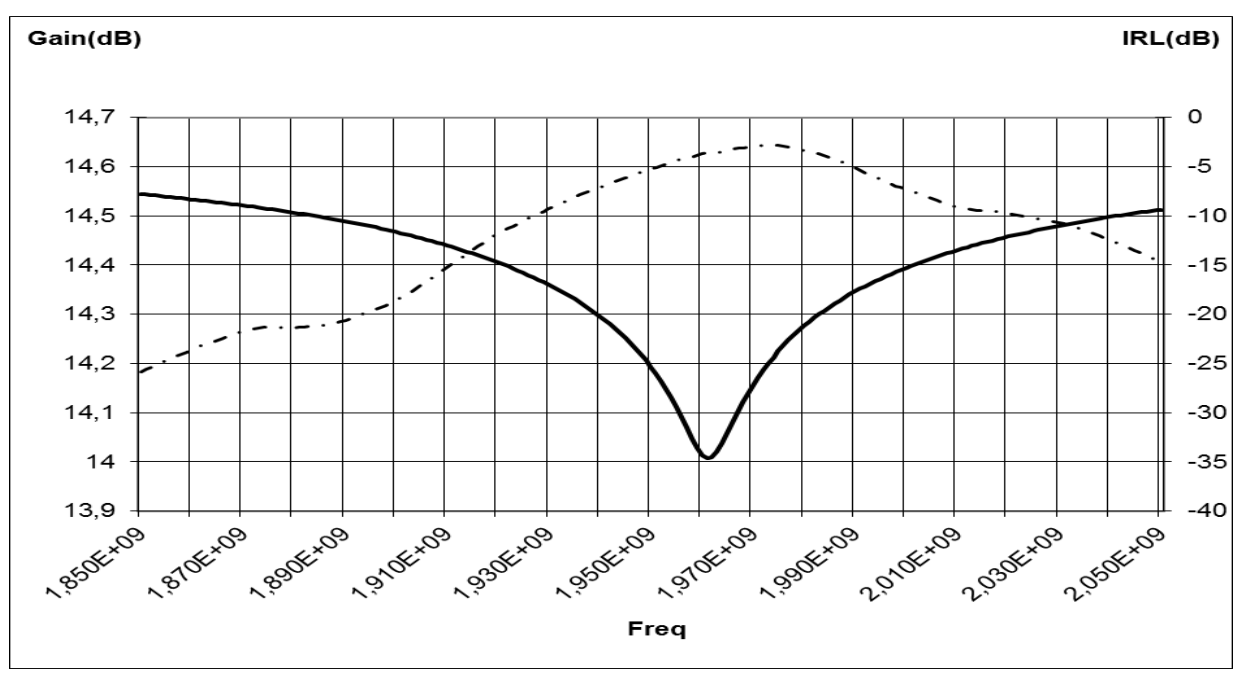

Fig. 22. Frequency sweep measurements for the MRF6S18100 LDMOS device.

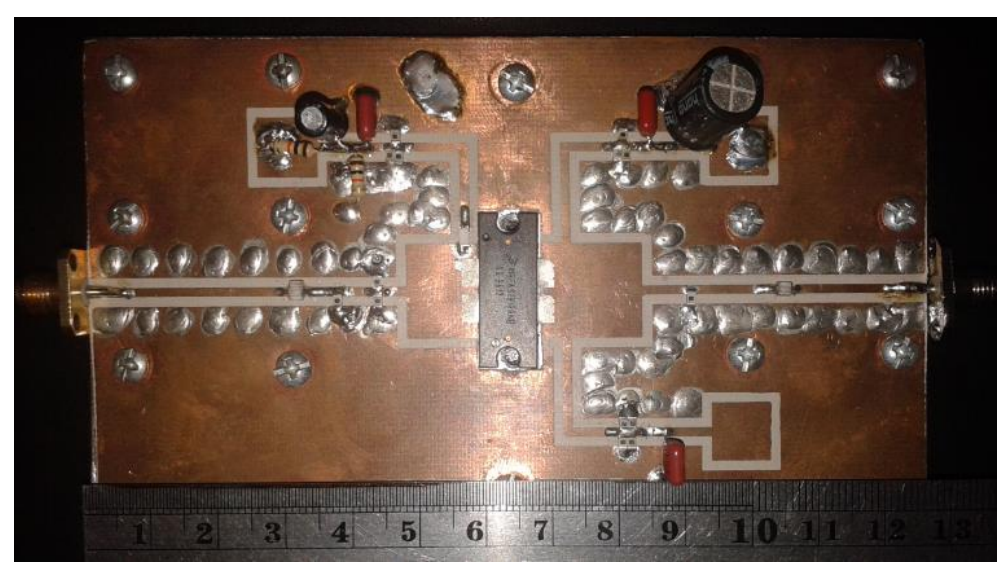

Fig. 23. Final prototype: implemented power amplifier using the MRF6S18100 LDMOS device.

\section{CONCLUSIONS}

In this paper we presented a methodology for modeling, design and implementation of amplifiers in different technologies. The first step is the modeling process, in which we used effective voltage equations to model both static and dynamic performance of the devices in multibias conditions. The advantage of this technique is that we can embed those equations into any conventional model such as COBRA, Angelov, Materka, Curtice, ROOT, MET, etc; and it is valid for different technologies such as GaAs, GaN, LDMOS, etc. In fact, beginning from static measurements (DC) we can set specific points for pulsed measurements (dynamic behavior) in order to optimize a nonlinear model for the 
microwave transistor. After that, by doing S-parameters measurements we can see the RF performance of the model which can be optimized in order to predict the gm/gds dispersion effect.

The second step is the substrate characterization in order to get accurate values for dielectric constant and loss tangent. In this case we demonstrated that we can measure them by using only 1port measurement and low cost equipment, while traditional methods uses 2 port measurements and VNAs. Finally, we designed a LDMOS power amplifier by using loadpull data, in a RF35 substrate previously characterized. By using symmetrical bias networks and lumped/discrete element combinations for the matching networks, we demonstrated that we can reach the appropriate impedances and satisfy the power and gain specs.

\section{ACKNOWLEDGMENT}

The authors thank Dr. Apolinar Reynoso- Hernández from CICESE (México) for the measurements on the GaN device. The authors also thank the design team and modeling team of Freescale Semiconductors, Toulouse, France (now NXP) for his valuable support with the LDMOS device.

\section{REFERENCES}

[1] Lan and al., "High power density InGaP PHEMTs for 26V operation", 2005 IEEE RFIC Symp. Digest.

[2] GaN HEMT equivalent circuit model with novel approach to dispersion modelling. Justin B. King; Thomas J. Brazil. 2012 7th European Microwave Integrated Circuit Conferenc

[3] Parker1 A. E. Parker, and J.G. Rathmell, "Bias and frequency dependence of FET characteristics," IEEE Trans. Microwave Theory and Tech., vol. 51, no.2, pp. 558-592, December 1997

[4] Single function drain current model for MESFET/HEMT devices including pulsed dynamic behavior. Guillermo Rafael-Valdivia; Ronan Brady; Thomas J. Brazil. 2006 IEEE MTT-S International Microwave Symposium Digest

[5] IMOC Nonlinear device model for GaN and GaAs microwave transistors including memory effects. G. RafaelValdivia, Anthony Urquizo, Thalia Mendoza. Silvio E. Barbin 2015 SBMO/IEEE MTT-S International Microwave and Optoelectronics Conference (IMOC)

[6] Improved prediction of the intermodulation distortion characteristics of MESFETs and PHEMTs via a robust nonlinear device model. V. I. Cojocaru; T. J. Brazil.1998 IEEE MTT-S International Microwave Symposium Digest

[7] Basque Country University. Physics of Materials Department. "Impedancia. Permitividad eléctrica". Available: http://www.sc.ehu.es/sqwpolim/FISICAII/P2.pdf

[8] JMOE Non-resonant permittivity measurement methods. Sergio L. S. Severo, Álvaro A. A. de Salles; Bruno Nervis, Braian K. Zanini. Journal of Microwaves, Optoelectronics and Electromagnetic Applications, Vol. 16, No. 1, March 2017

[9] "FR4" Available: http://www.farnell.com/datasheets/1644697.pdf

[10] Chen Wang, "Determining Dielectric Constant and Loss tangent in FR-4", UMR EMC. Laboratory technical report: TR00-1-041,. March (2000). Available: http://emclab.mst.edu/documents/TR00-1-041.pdf

[11] RF35. Available: http://www.taconic.co.kr/english/download/RF-35.pdf

[12] Datasheet. Available: http://www.datasheet.hk/view_download.php?id=1127867\&file=0068 $\mathrm{mrf6ss18100n \_ 549062.pdf}$

[13] J.M. Coupat and al., "True pulse load pull measurement setup for high power transistors characterization", 2006 ARFTG Conference.

[14] "A 3.5 GHz 25 W Silicon LDMOS RFIC power amplifier for WiMAX applications" C. Cassan, P. Gola Freescale Semiconductors, inc., RF division RFIC Honolulu June 3th-5th 2007 International Microwave Symposium. 